\title{
The association among leukocyte apoptosis, autoantibodies and disease severity in systemic lupus erythematosus
}

\author{
Yu-Jih Su ${ }^{1,6}$, Tien-Tsai Cheng ${ }^{1}$, Chung-Jen Chen ${ }^{1}$, Wen-Chan Chiu', Chung-Yuan Hsu' ${ }^{1}$, Wen-Neng Chang ${ }^{2}$, \\ Nai-Wen Tsai ${ }^{2}$, Chia-Te Kung ${ }^{3}$, Hung-Chen Wang ${ }^{4}$, Wei-Che Lin ${ }^{5}$, Chih-Cheng Huang ${ }^{2}$, Ya-Ting Chang ${ }^{2}$, \\ Chih-Min Su, ${ }^{6,3}$, Yi-Fang Chiang ${ }^{2}$, Ben-Chung Cheng ${ }^{1,6}$, Yu-Jun Lin ${ }^{6,4}$ and Cheng-Hsien Lü ${ }^{2,6^{*}}$
}

\begin{abstract}
Background: Both apoptosis and autoantibodies are important factors associated with disease activity in the pathogenesis of systemic lupus erythematosus (SLE). This study tested the hypothesis that increased leukocyte apoptosis is associated with elevated levels of autoantibodies and the disease activity of SLE.

Methods: Leukocyte apoptosis was determined by flow cytometry, including annexin V, APO2.7, and 7-amino-actinomycin D (7-AAD) on each subtype of leukocyte in 23 patients with SLE. Leukocyte apoptosis was also evaluated in nine patients with Sjogren's syndrome (SJS) and in 20 volunteer subjects. Titers of common autoantibodies and the disease activity index (SLEDAI-2 k) of the SLE patients were also determined.

Results: Except for annexin $V$ and APO 2.7 of monocytes and late apoptosis (annexin $V+7$-ADD) of lymphocytes, apoptosis in the total and in subsets of leukocytes were significantly higher in SLE patients than in controls (all $p<0.05$, post hoc analysis). The mean percentage of late apoptosis of leukocytes (annexin $V+7-A A D)$ positively correlated with levels of anti-Ro52/60 ( $r=0.513, p<0.01)$, anti-La $(r=0.439, p=0.04)$, and anti-Mi-2 $(r=0.492$, $p=0.02$ ), and inversely correlated with both $C 3$ and $C 4$ levels, although not statistically significant. The percentage of APO2.7 of CD19+ cells positively correlated with SLEDAl-2 K score $(p=0.01)$.
\end{abstract}

Conclusions: Leukocyte apoptosis is significantly higher in patients with SLE and correlates well with the levels of several autoantibodies. The APO2.7 of B-lymphocyte (CD19+) cells positively correlates with the disease activity of SLE.

Keywords: Autoantibodies, Diseases severity score, Leukocyte apoptosis, Systemic lupus erythematosus

\section{Introduction}

Systemic lupus erythematosus (SLE) is a chronic systemic disease affecting mostly women of child-bearing age. It is the prototype of autoimmune diseases because of the variety of proposed pathogenesis. Dozens of autoantibodies are linked with SLE patients, including some that are diagnostic and specific [1-3], such as anti-double stranded deoxyribonucleic acid antibody (a-dsDNA), anti-Smith antibody (a-sm), and anti-ribosomal P (a-rib p). The disease

\footnotetext{
*Correspondence: chlu99@ms44.url.com.tw

${ }^{2}$ Department of Neurology, Chang Gung Memorial Hospital, 123, Ta Pei

Road, Niao Sung Hsiang, Kaohsiung, Taiwan

${ }^{6}$ Department of Biological Science, National Sun Yat-Sen University,

Kaohsiung, Taiwan

Full list of author information is available at the end of the article
}

activity of SLE has also been associated with these autoantibodies.

Apoptosis is one of several important mechanisms involved in the pathogenesis of SLE. A previous study has demonstrated that lymphocyte apoptosis is increased and removal of apoptotic cells is impaired in SLE. Furthermore, apoptosis is higher in active SLE compared to inactive SLE [4].

Several lines of evidence demonstrate the close relationship of apoptosis and autoantibodies. Delayed clearance of apoptotic residue, such as histone H3 [5], may result in the activation of antigen-presenting cells and the formation of autoantibodies [6,7]. The antigenicity of autoantibodies is mostly against apoptotic cells, which

\section{Biomed Central}

(c) 2013 Su et al.; licensee BioMed Central Ltd. This is an open access article distributed under the terms of the Creative Commons Attribution License (http://creativecommons.org/licenses/by/2.0), which permits unrestricted use, distribution, and reproduction in any medium, provided the original work is properly cited. 
has been demonstrated by Huggins et al. [8]. Most studies link lymphocyte apoptosis with titers of autoantibodies $[9,10]$, but little is mentioned regarding leukocyte apoptosis. The present study focuses on SLE patients due to this lack of detailed data.

To date, little is known about the relationship among leukocyte apoptosis, levels of autoantibodies, and severity of SLE. With the hypothesis that increased leukocyte apoptosis is associated with elevated levels of autoantibodies and disease activity of SLE, this prospectively-designed study aimed to report on the relationship among percentage of leukocyte apoptosis, levels of autoantibodies, and SLE disease activity.

\section{Patients and methods}

\section{Study patients}

Twenty-three patients with definitive diagnosis of SLE and followed-up at the Rheumatology Outpatient Clinic for more than 6 months were prospectively evaluated. The diagnostic criteria for SLE were based on the 1997 revision of 1982 American College of Rheumatology (ACR) classification criteria for SLE [3], while the clinical assessment of disease activity of SLE was according to the SLE disease activity index (SLEDAI-2 K) [11]. For comparison, 20 age- and sexmatched healthy subjects year were enrolled as healthy controls and nine patients with Sjogren's syndrome (SJS) were the disease controls. The SJS patients were diagnosed based on classification criteria for SJS [12].

The Institutional Review Committee on Human Research approved the study protocol and the participants provided informed consent. Patients were excluded if they had autoimmune diseases other than SLE or SJS, or if they had fever or any infectious disorder that could affect the WBC count.

\section{Clinical assessments}

All of the subjects underwent complete medical examinations upon enrollment. Biomarkers were also collected. Since there were no clinical practice guidelines for SLE flare-up, the important decision in immuno-suppressant adjuvant treatment was whether or not to add a cytotoxic or corticosteroid-sparing drug like cyclophosphamide, methotrexate, azathioprine, mycophenolate mofetil, or leflunomide. Severe SLE was defined as an SLEDAI-2 K score $>12$, while mild-to-moderate SLE was an SLEDAI-2 K score $\leq 12$ [13].

\section{Blood sampling and assessment of leukocyte apoptosis}

Blood samples were collected by venipuncture of the patient's forearm vein.

\section{Flow cytometry assay using APO 2.7 Antibody for detecting apoptosis}

All of the flow cytometry assays were performed within one hour after blood extraction to ensure that the results were as close as possible to the in vivo situation. Fixed amounts of blood were diluted 1:5 with PBS, and $100 \mu \mathrm{L}$ was stained with $10 \mu \mathrm{L}$ of each of the following: fluorescence conjugated monoclonal antibodies against CD45-phycoerythrin (PE)-Cy5 (clone J33), CD61-fluorescein isothiocyanate (FITC; clone SZ21), and APO 2.7-PE (clone 2.7A6A3; Immunotech, Marseille, France). These were then titrated at saturating concentrations.

The CD45-PE-Cy5 antibody reacted with the CD45 family of trans-membrane glycoproteins, expressed on the surface of all human leukocytes, and a pan-leukocyte marker. The CD61-FITC antibody was a pan-platelet marker that reacted with the GPIIb/IIIa complex (fibrinogen receptor). The APO 2.7-PE antibody reacted with a $38-\mathrm{kDa}$ mitochondrial membrane protein (7A6 antigen), which was detectable on non-permeabilized cells in the late apoptotic state [14].

Annexin V staining, relevant to early apoptosis, produced similar results but was rejected for questionable reliability under fixation conditions, with formaldehyde clearly biasing the staining results. Mouse immunoglobulin G-PE was a control for non-specific staining, which did not differ from the APO2.7-PE signal on platelets, such that each subject was its own control without changing the sample tube. After $30 \mathrm{~min}$ of incubation in the dark at room temperature, the stained samples were diluted with $0.5 \mathrm{ml}$ of FACSFlow (Becton Dickinson, San Jose, CA).

Flow cytometry analysis was performed immediately after staining using an Epics XL flow cytometer (Beckman Coulter, Fullerton, California) and the CellQuest software. Five thousand CD45-PE-Cy5+ cells per sample were acquired in a combined forward and side scatters and deepred FL4 fluorescence (CD45-PE-Cy5) leukocyte gate. Another 5000 CD61-FITC + cells per sample were acquired in a combined forward and side scatters and green FL1 fluorescence (CD61-FITC) platelet gate to define a negative control threshold for the measurement of apoptosis, such that each subject was its own control.

\section{Annexin V-FITC 7-AAD fluorescence-activated cell analysis}

Membrane phosphatidyl-serine was detected by annexin- $V$ using a commercially available kit (Boehringer Mannheim, Indianapolis, Indiana). The PBS-washed leucocytes were incubated with annexin V-FITC and 7-amino-actinomycin D (7-AAD) for $15 \mathrm{~min}$ at room temperature according to manufacturer's guidelines. Samples were transferred to 5-mL polypropylene tubes, diluted $900 \mu \mathrm{L}$ with Hanks' balanced salt solution, and placed on ice before analysis by flow cytometry. The samples were analyzed using an Epics 
XL flow cytometer (Beckman Coulter, Fullerton, California) and the CellQuest software. Fifteen thousand events were counted per sample. Low-fluorescence debris was gated out of the analysis. Leukocyte subtypes were identified according to their CD45 expression intensity and divided into neutrophils, monocytes, and lymphocytes. From here on, white blood cells (WBC) represented total leukocytes.

Annexin V-FITC staining was identified in fluorescent-1 and 7-AAD staining in fluorescent-4. Cells were identified as follows: early apoptotic cells if they were positive for marker annexin V-FITC but negative for 7-AAD; late apoptotic cells if they were positive for annexin V-FITC and 7-AAD; dead cells if they were negative for annexin V-FITC but positive for 7-AAD; and viable cells if they were negative for annexin V-FITC and 7-AAD.

\section{Apoptosis of lymphocytes}

Fixed amounts of blood were diluted 1:5 with PBS and $100 \mu \mathrm{L}$ was stained with $10 \mu \mathrm{L}$ of each of the following: fluorescence conjugated monoclonal antibodies against CD4-phycoerythrin (PE)-Cy5, CD19-fluorescein isothiocyanate (FITC), and CD8- phycoerythrin (PE). Each of the above staining was further stained with annexin $\mathrm{V}$ FITC, 7-amino-actinomycin D (7-AAD), or APO 2.7-PE (clone 2.7A6A3; Immunotech, Marseille, France). These were then titrated at saturating concentrations. Samples were transferred to 5-mL polypropylene tubes, diluted with $900 \mu \mathrm{L}$ of Hanks' balanced salt solution, and placed on ice before flow cytometry. Samples were analyzed using an Epics XL flow cytometer (Beckman Coulter, Fullerton, California) and the CellQuest software. Fifteen thousand events were counted per sample. Lymphocyte subtypes were identified according to their surface antigen (i.e., CD4, CD8, or CD19) expression intensity. A database coordinator was responsible for monitoring all data collection and entry.

\section{Autoantibody detection}

Autoantibody titers, including IgG antibodies against dsDNA (a-dsdna), Ribosomal p (a-ribp), Ro (52/60) (a-ro), Ro $52 \mathrm{kDa}$ (a-ro52), Ro $60 \mathrm{kDa}$ (a-ro60), La (a-la), U1RNP (a-u1rnp), Sm (a-sm), and Mi-2 (a-mi2) were detected by Elia-Phadia 250 according to the operation manual. All data were checked for any inconsistencies. Intra-assay variability based on repeated measurements of the same blood sample was low, with mean coefficients of variance between $6.11 \%$ and $14.28 \%$ for SLE patients, and $6.0 \%$ in control individuals.

\section{Statistical analysis}

Data were expressed as mean $\pm \mathrm{SD}$ or median (inter-quartile range). Categorical variables were compared using Chi-square test or Fisher's exact test, as appropriate. The percentage of leukocyte apoptosis and autoantibody level were logarithmically transformed to improve normality. Univariate analyses were compared using Student's t-test. Continuous variables among the three groups (SLE, SIS, and volunteer control) were compared using one-way ANOVA, followed by Bonferroni post hoc multiple comparison procedure. Correlation analysis was used to evaluate the relationship among mean SLEDAI-2 K score, percentage of leukocyte apoptosis, and levels of autoantibodies. Statistical significance was set at $p<0.05$. Receiver operating characteristic (ROC) curves were generated for leukocyte apoptosis. The areas under the ROC curves (AUC) were calculated for each parameter and compared. All statistical calculations were performed using the SAS software package, version 9.1 (2002, SAS Statistical Institute, North Carolina).

\section{Results}

\section{Baseline characteristics of the study patients}

The baseline characteristics and laboratory data of SLE patients, SJS patients, and control groups, as well as their medications, were listed in Table 1. Although the mean age was older in the SIS group, there was no statistically significant difference in age and sex among the three groups ( $p=0.16$ and $p=0.541$, respectively). The clinical symptoms of the 23 SLE patients included musculoskeletal involvement in 15, neurologic involvement in 14, muco-cutaneous involvement in two, hematologic involvement in six, cardio-respiratory involvement in two, and renal involvement in two. The clinical symptoms of the nine patients with SJS were also listed.

\section{Leukocyte apoptosis in patients with SLE, patients with SJS, and the controls}

The laboratory data and percentage of leukocyte apoptosis of the three groups were listed in Table 2. Except for hemoglobin level, the WBC and platelet counts were not significantly different among the three groups. Except for annexin V and APO2.7 in monocytes and annexin V+ 7AAD in lymphocytes, the percentages of apoptosis of total leukocytes and the leukocyte subsets were significantly higher in the SLE patients than in the controls.

\section{Correlation of clinical severity, autoantibody level, and percentage of leukocyte apoptosis}

Regarding the innate immune cells, the mean percentages of late apoptosis of leukocyte (annexin V-FITC and 7-AAD) positively correlated with levels of anti-dsDNA $(r=0.415, p<0.05)$, anti-Ro52/60 $(r=0.513, p<0.01)$, anti-La $(r=0.439, p=0.04)$, anti-Mi-2 $(r=0.492, p=0.02)$, and inversely correlated with both $\mathrm{C} 3$ and $\mathrm{C} 4$ levels, although not statistically significant (Table 3 ). The SLE disease activity index (SLEDAI-2 K) positively correlated with anti-Ro $60 \mathrm{kDa}(r=0.407, p=0.05)$, but negatively correlated with complement C4 level $(r=-0.508, p=0.01)$. 
Table 1 Baseline characteristics of SLE patients, SJS patients, and control subjects

\begin{tabular}{|c|c|c|c|}
\hline & $\begin{array}{l}\text { SLE patients } \\
\qquad(\mathrm{n}=23)\end{array}$ & $\begin{array}{l}\text { Sjogren's syndrome } \\
\qquad(\mathrm{n}=9)\end{array}$ & $\begin{array}{l}\text { Healthy controls } \\
\qquad(n=20)\end{array}$ \\
\hline Age $(y)($ mean \pm SD) & $44.38 \pm 10.87$ & $56.56 \pm 13.86$ & $48.37 \pm 12.01$ \\
\hline Male /female & $2 / 21$ & $1 / 8$ & $6 / 14$ \\
\hline Clinical symptoms & + & & \\
\hline Constitutional & 9 & 0 & - \\
\hline Muco-cutaneous involvement & 2 & 0 & - \\
\hline Neurological involvement & 14 & 7 & - \\
\hline Musculo-skeletal involvement & 15 & 2 & - \\
\hline Cardio-respiratory involvement & 2 & 0 & - \\
\hline Renal involvement & 3 & 0 & - \\
\hline Hematologic involvement & 6 & 0 & - \\
\hline Median (IQR) SLEDAI-2 K & $11(6-14)$ & & - \\
\hline Mean dosage of medication (mg/day) & $\xi$ & $g$ & \\
\hline Prednisolone & 21 (mg/day) $(n=15)$ & 3.3 (mg/day) $(n=5)$ & - \\
\hline Hydroxychloroquine & 300 (mg/day) $(n=17)$ & 150 (mg/day) $(\mathrm{n}=5)$ & - \\
\hline Azathioprine & $50(\mathrm{mg} /$ day $)(\mathrm{n}=1)$ & 0 & - \\
\hline Mycophenolate & $1080(\mathrm{mg} /$ day $)(\mathrm{n}=1)$ & 0 & - \\
\hline Cyclophosphamide & 500 (mg/month) $(\mathrm{n}=2)$ & 0 & - \\
\hline Cyclosporine & 50 (mg/day) $(\mathrm{n}=3)$ & 0 & - \\
\hline
\end{tabular}

${ }^{\dagger} 17$ patients who had more than one clinical symptom.

$\varepsilon 12$ patients who took more than one medication.

$9_{4} 4$ patients who took more than one medication.

Abbreviations: SLE Systemic lupus erythematosus, SJS Sjogren's syndrome, IQR Inter-quartile range, SLEDAI-2 K Systemic lupus erythematosus disease activity index 2000.

Regarding the adaptive immune cells, the percentage of CD4 (+) cell apoptosis in APO2.7 negatively correlated with anti-Ro $52 \mathrm{kDa}(r=-0.717, p=0.03)$, and anti-Ro52/60 $(r=-0.684, p=0.04)$. The percentage of CD19 $(+)$ cell apoptosis in APO2.7 positive correlated with SLE disease activity index (SLEDAI-2 K) $(r=0.793, p=0.01)$, but negative correlated with a-dsDNA $(r=-0.883, p<0.001)$. Furthermore, the AUC for the percentage of APO2.7 in B-lymphocyte (CD19+) apoptosis was 0.95 ( $p=0.027$, 95\% CI: 0.00-1.00). The cut-off value of the percentage of APO2.7 in B-lymphocyte (CD19+) apoptosis for severe SLE was $1.01 \%$ (sensitivity $75 \%$ and specificity $95 \%$ ). Other results were listed in Table 3.

\section{Discussion}

The present study has several major findings. First, the percentage of leukocyte apoptosis (including neutrophil, monocyte, and lymphocyte apoptosis) is significantly higher in SLE patients than in healthy controls. Second, the mean percentages of late apoptosis of leukocyte (annexin $\mathrm{V}+7$-AAD) positively correlate with levels of most common autoantibodies (e.g. anti-Ro52/60, anti-La, and anti-Mi-2). Third, the percentage of CD4 (+) cell apoptosis in APO2.7 significantly but negatively correlates with levels of several autoantibodies (e.g. anti-Ro52/60, and anti-Ro52kDa). Fourth, the level of C3 negatively correlates with the late apoptosis of granulocyte (7$\mathrm{AAD}+$ annexin $\mathrm{V})$. The level of $\mathrm{C} 4$ positively correlates with the late apoptosis (7-AAD + annexin V) of CD8 cells. Lastly, the percentage of APO2.7 on B-lymphocyte (CD19+) apoptosis significantly correlates with median SLEDAI-2K score. The cut-off value of $1 \%$ of APO2.7 on B-lymphocyte (CD19+) apoptosis is associated with severe SLE. APO2.7 is expressed on the mitochondrial membrane during early apoptosis in relation to the release of cytochrome $c$ and represents the activationinduced cell death under certain circumstances $[15,16]$.

Several lines of blood cells have defects in the apoptosis pathway in SLE patients, either prone or resistant to apoptosis [17-23]. Some studies reveal increased monocyte and lymphocyte apoptosis [18,20,21,23], but other studies demonstrate increased leukocyte apoptosis [22], and decreased B-lymphocyte apoptosis [17] or no difference in apoptosis [19]. Generally speaking, in the present study, the percentages of apoptosis in most leukocyte subsets, including neutrophils, monocytes, and lymphocytes, are significantly higher in SLE patients than in controls.

Leukocyte late apoptosis markers are positively correlated with levels of several autoantibodies, including 
Table 2 Laboratory data of the SLE patients and control subjects

\begin{tabular}{|c|c|c|c|c|}
\hline & $\begin{array}{l}\text { SLE patients } \\
\quad(n=23)\end{array}$ & $\begin{array}{l}\text { SJS patients } \\
\quad(n=9)\end{array}$ & $\begin{array}{l}\text { Healthy controls } \\
\qquad(n=20)\end{array}$ & $\begin{array}{c}p \\
\text { value }\end{array}$ \\
\hline White blood cells $(\times 103 / \mathrm{ml})$ & $6.73 \pm 2.5$ & $6.47 \pm 1.97$ & $5.68 \pm 1.89$ & 0.442 \\
\hline$\%$ granulocyte & $69.49 \pm 13.19$ & $65.93 \pm 10.22$ & $61.6 \pm 12.76$ & 0.341 \\
\hline \% lymphocyte & $22.81 \pm 11.65$ & $25.33 \pm 9.15$ & $31.01 \pm 10.85$ & 0.232 \\
\hline$\%$ monocyte & $5.57 \pm 3.00$ & $6.19 \pm 2.44$ & $5.63 \pm 1.74$ & 0.873 \\
\hline Hemoglobulin (mg/dL) & $11.73 \pm 1.73$ & $12.38 \pm 0.99$ & $14.06 \pm 1.86$ & $0.003^{a}$ \\
\hline Platelet counts (×104/ml) & $22.13 \pm 8.49$ & $23.67 \pm 5.39$ & $22.26 \pm 6.19$ & 0.851 \\
\hline \multicolumn{5}{|l|}{ Autoantibodies titers } \\
\hline a-dsdna & $18(1.7,70.0)$ & ND & ND & ND \\
\hline a-ro & $0.5(0.1,240)$ & $84.75(0.3,217.5)$ & ND & 0.725 \\
\hline a-ro52 & $0.3(0.1,32)$ & ND & ND & ND \\
\hline a-ro60 & $0.9(0,300)$ & ND & ND & ND \\
\hline a-la & $0.2(0,3.3)$ & $0.2(0.1,4.45)$ & ND & 0.896 \\
\hline a-mi2 & $0.1(0,0.2)$ & ND & ND & ND \\
\hline \multicolumn{5}{|c|}{ Apoptosis tested by flow cytometry? } \\
\hline \multicolumn{5}{|l|}{ Leukocyte apoptosis (\%) } \\
\hline Annexin V (\%) & $15.3(8.9-18.2)$ & $9.87(8.29-12.50)$ & $10.9(8.8-13.4)$ & $0.005^{\beta}$ \\
\hline APO2.7 (\%) & $1.3(0.9-2.2)$ & $1.10(0.70-1.57)$ & $0.7(0.5-0.9)$ & $0.001^{Y}$ \\
\hline Annexin V + 7AAD (\%) & $7.1(3.8-11.8)$ & $6.58(2.80-10.10)$ & $3.2(2.6-4.5)$ & $0.011^{\delta}$ \\
\hline \multicolumn{5}{|l|}{ Neutrophil apoptosis (\%) } \\
\hline Annexin V (\%) & $21.6(11.7-28.8)$ & $9.15(7.80-15.76)$ & $13.9(10.3-18.0)$ & $0.010^{\varepsilon}$ \\
\hline APO2.7 (\%) & $0.6(0.4-1.3)$ & $0.79(0.40-1.05)$ & $0.5(0.4-0.6)$ & $0.001^{\zeta}$ \\
\hline Annexin V + 7AAD (\%) & $10.7(3.1-22.8)$ & $6.93(1.97-20.60)$ & $3.9(2.2-5.5)$ & $0.026^{n}$ \\
\hline \multicolumn{5}{|l|}{ Lymphocyte apoptosis (\%) } \\
\hline Annexin V (\%) & $8.4(4.9-12.1)$ & $6.10(5.11-8.23)$ & $4.8(3.8-6.7)$ & $0.009^{\ominus}$ \\
\hline APO2.7 (\%) & $0.7(0.5-1.0)$ & $0.46(0.43-0.78)$ & $0.3(0.2-0.4)$ & $0.008^{k}$ \\
\hline Annexin V + 7AAD (\%) & $2.1(1.2-3.6)$ & $1.46(0.67-2.92)$ & $1.5(0.9-2.1)$ & 0.101 \\
\hline \multicolumn{5}{|l|}{ Monocyte apoptosis (\%) } \\
\hline Annexin V (\%) & $19.6(13.5-27.3)$ & $13.83(11.42-15.38)$ & $17.4(12.4-21.6)$ & $0.032^{\lambda}$ \\
\hline APO2.7 (\%) & $3.0(2.5-6.8)$ & $1.94(1.48-3.27)$ & $1.8(1.1-2.7)$ & 0.088 \\
\hline Annexin V + 7AAD (\%) & $10.4(5.3-17.8)$ & $7.63(3.34-15.36)$ & $4.6(3.3-6.0)$ & $0.024^{\mu}$ \\
\hline
\end{tabular}

Abbreviations: SLE Systemic lupus erythematosus, SJS Sjogren's syndrome, WBC White blood cells or leukocytes, 7-AAD 7-amino-actinomycin D, SD Standard deviation, IQR Inter-quartile range, A-dsdna Anti-dsDNA, A-ro Anti-Ro52/60, A-ro52 Anti-Ro 52 kDa, A-ro60 Anti-Ro 60 kDa, A-la Anti-La, ND Not done.

${ }^{\dagger}$ Figures are median (IQR) or number (\%).

Results of Bonferroni multiple comparisons for post hoc test (SLE vs. control, SLE vs. SJS, or SJS vs. control).

$a$, SLE vs. control $=0.002 ; \beta$, SLE vs. control $=0.014$, SLE vs. SJS $=0.029 ; \gamma$, SLE vs. control $=0.001 ; \delta$, SLE vs. control $=0.009 ; \varepsilon, \operatorname{SLE}$ vs. control $=0.005$, SLE vs.

$\mathrm{SJS}=0.004 ; \zeta$, SLE vs. control $=0.041 ; \eta$, SLE vs. control $=0.021 ; \theta$, SLE vs. control $=0.008 ; \mathrm{k}, \mathrm{SLE}$ vs. control $=0.007 ; \lambda, \mathrm{SLE}$ vs. $\mathrm{SJS}=0.036 ; \mu, \mathrm{SLE}$ vs. control $=0.026$.

anti-Ro, anti-La, and anti-Mi2. This is an evident link between leukocyte apoptosis and antibody formation, as previously proposed $[24,25]$.

The CD4 (+) cell apoptosis marker APO2.7 is negatively correlated with levels of anti-Ro52, and anti-Ro. Since the anti- Ro antibody is elevated in many patients with lupus [26], CD4 (+) cell apoptosis may be decreased in SLE patients. The present study demonstrates that the APO2.7 of CD19+ lymphocytes is positively correlated with disease activity index. Several studies have demonstrated that the appearance of APO2.7 on lymphocytes represents activation and elevated titers of autoantibodies [16,27]. However, activated APO2.7 on lymphocytes may be reversed by downstream anti-apoptosis molecules, as shown in other studies [15,28]. Lastly, increased activated APO2.7 of CD19+ lymphocytes may undergo apoptosis after immuno-suppressant therapy [29]. Thus, in this study, activation through a mitochondria-dependent pathway among CD19+ lymphocytes is prominent in patients with active SLE. Disease activity is positively correlated 
Table 3 Correlation among apoptotic markers, disease activity, and levels of autoantibodies in lupus patients

\begin{tabular}{cccccccccc}
\hline & & SLEDAI2k & c3 & c4 & a-ro & a-ro52 & a-ro60 & a-la & a-mi2 \\
\hline (7AAD + annexinV) of WBC (\%) & $r$ & 0.14 & -0.3 & -0.19 & $.513^{*}$ & 0.29 & 0.41 & $.439^{*}$ & $.492^{*}$ \\
& $p$ value & 0.54 & 0.17 & 0.39 & 0.01 & 0.17 & 0.05 & 0.04 & 0.02 \\
(7AAD + annexinV) of Granulocyte (\%) & $r$ & 0.18 & $-.460^{*}$ & -0.2 & 0.33 & 0.2 & 0.32 & 0.33 & 0.3 \\
& $p$ value & 0.42 & 0.03 & 0.37 & 0.13 & 0.36 & 0.13 & 0.13 & 0.16 \\
(7AAD + annexinV) of CD8 (+)(\%) & $r$ & -0.06 & 0.35 & $.636^{*}$ & -0.07 & 0.07 & -0.21 & -0.09 & -0.11 \\
& $p$ value & 0.87 & 0.33 & 0.04 & 0.85 & 0.85 & 0.57 & 0.8 & 0.77 \\
APO2.7 of CD4(+)(\%) & $r$ & 0.3 & 0.17 & 0.05 & $-.684^{*}$ & $-.717^{*}$ & -0.62 & -0.57 & -0.24 \\
& $p$ value & 0.43 & 0.67 & 0.9 & 0.04 & 0.03 & 0.08 & 0.11 & 0.54 \\
APO2.7 of CD19 (+)(\%) & $r$ & $.793^{*}$ & -0.45 & -0.48 & 0.32 & 0.34 & 0.29 & 0.4 & -0.07 \\
SLEDAl2k & $p$ value & 0.01 & 0.22 & 0.19 & 0.4 & 0.37 & 0.45 & 0.28 & 0.86 \\
& $r$ & $x$ & -0.35 & $-.508^{*}$ & 0.3 & 0.23 & $.407^{*}$ & 0.24 & 0.21 \\
& $p$ value & & 0.1 & 0.01 & 0.15 & 0.29 & 0.05 & 0.25 & 0.32 \\
\hline
\end{tabular}

Note: ${ }^{*}, \mathrm{p}<0.05$.

Abbreviations: $R$ Correlation coefficient, A-ro Anti-Ro52/60, A-ro52 Anti-Ro 52 kDa, A-ro60 Anti-Ro 60 kDa, A-la Anti-La, A-mi2 Anti-Mi-2, WBC White blood cells or leukocytes, 7-AAD 7-amino-actinomycin D, SLEDAl-2 K Systemic lupus erythematosus disease activity index 2000.

with anti-Ro60 titers in this study, as previously mentioned in other investigations $[1,2]$.

During SLE flare-up, both C3 and C4 are decreased, so the late apoptosis of granulocyte increases [30] but the apoptosis of CD8 (+) cells decreases. The present study also demonstrates that the level of $\mathrm{C} 3$ is significantly but negatively correlated with the late apoptosis of granulocyte (7-AAD + annexin V), while the level of $\mathrm{C} 4$ is positively correlated with the late apoptosis (7-AAD + annexin $\mathrm{V}$ ) of CD8 cells (Table 3). Linker-Israeli et al. have shown that CD8 (+) cells participate in autoantibody synthesis in SLE [31].

There are significant differences of apoptosis markers in annexin V of leukocytes (SLE vs. SJS, $p=0.029$ ), neutrophils (SLE vs. SJS, $p=0.004$ ), and monocytes (SLE vs. SJS, $p=0.036)$ during the post hoc analysis, despite no difference in absolute numbers of leukocytes, neutrophils, and monocytes between SLE and SIS patients. Similarly, Armstrong et al [22] demonstrated higher annexin $\mathrm{V}$ of neutrophils among SLE patients compared to those with other autoimmune diseases, while Bengtsson et al [21] demonstrated higher annexin $\mathrm{V}$ of monocytes. These finding suggest that there are more autoantigens, which may the induce apoptosis of leukocytes, in SLE patients than in the disease controls.

This study has several limitations. First, there are many assays for evaluating apoptosis in vitro so the results and interpretations from various methodologies may be partially different. The level of apoptosis tested by flow cytometry may not necessarily reflect the real leukocyte physiologic function in vivo. Similar protocols are used to detect cell apoptosis $[29,32,33]$. Second, this is a crosssectional observational study. Theoretically, high-dose immuno-suppressant drugs can affect leukocyte activity and autoantibody levels. Although the dosages of steroids (mean, $31 \mathrm{mg} /$ day) and hydroxychloroquine (mean, $300 \mathrm{mg} /$ day) used in this case control study are not high, their effects still cannot be excluded. Lastly, the case number in this study is not large and similar aspects of results have been obtained from other series studies with small case numbers [34,35]. Large-scale prospective and longitudinal studies are needed to evaluate the prognostic contribution of leukocyte apoptosis on clinical outcome.

In conclusion, leukocyte apoptosis is significantly higher in SLE patients and correlates well with the levels of several autoantibodies. The APO2.7 of B-lymphocyte (CD19+) cells positively correlates with the disease activity of SLE.

\section{Ethical approval}

The study was approved by Chang Gung Memorial Hospital's Institutional Review Committee on Human Research.

\section{Competing interests}

The authors declare that they have no competing interests.

\section{Author contributions}

YJS participated in the study design and manuscript drafting. TTC, CJC, WCC, CYH, WNC, CTK, HCW, WCL, CCH, YTC, CMS, YFC, BCC, and YJL participated in the sequence alignment and clinical evaluation of patients. NWT performed the statistical analysis. CHL conceived the study, and participated in its design and coordination, and helped draft the manuscript. All authors read and approved the final manuscript.

\section{Acknowledgements}

This work was supported by grants from Chang Gung Memorial Hospital (Chang Gung Medical Research Project CMRPG8B1281) and NHRI-EX101-10142EI. The authors thank all of the subjects who participated in this study and wish to thank Dr. Gene Alzona Nisperos for editing and reviewing the manuscript for English language considerations. 


\section{Author details}

${ }^{1}$ Departments of Internal Medicine, Chang Gung Memorial Hospital-Kaohsiung Medical Center, Chang Gung University College of Medicine, Kaohsiung, Taiwan. ${ }^{2}$ Department of Neurology, Chang Gung Memorial Hospital, 123, Ta Pei Road, Niao Sung Hsiang, Kaohsiung, Taiwan. ${ }^{3}$ Emergency Medicine, Chang Gung Memorial Hospital-Kaohsiung Medical Center, Chang Gung University College of Medicine, Kaohsiung, Taiwan. ${ }^{4}$ Neurosurgery, Chang Gung Memorial Hospital-Kaohsiung Medical Center, Chang Gung University College of Medicine, Kaohsiung, Taiwan. ${ }^{5}$ Radiology, Chang Gung Memorial Hospital-Kaohsiung Medical Center, Chang Gung University College of Medicine, Kaohsiung, Taiwan. ${ }^{6}$ Department of Biological Science, National Sun Yat-Sen University, Kaohsiung, Taiwan.

Received: 5 July 2013 Accepted: 14 October 2013

Published: 19 October 2013

\section{References}

1. Hanly JG, et al: Comparison between multiplex assays for autoantibody detection in systemic lupus erythematosus. J Immunol Meth 2010, 358(1-2):75-80.

2. Hassan $A B$, et al: Serial analysis of Ro/SSA and La/SSB antibody levels and correlation with clinical disease activity in patients with systemic lupus erythematosus. Scand J Rheumatol 2002, 31(3):133-139.

3. Smith EL, Shmerling RH: The American college of rheumatology criteria for the classification of systemic lupus erythematosus: strengths, weaknesses, and opportunities for improvement. Lupus 1999, 8(8):586-595.

4. Jin O, et al: Lymphocyte apoptosis and macrophage function: correlation with disease activity in systemic lupus erythematosus. Clin Rheumatol 2005, 24(2):107-110.

5. van Bavel CC, et al: Apoptosis-induced histone $\mathrm{H} 3$ methylation is targeted by autoantibodies in systemic lupus erythematosus. Ann Rheum Dis 2011, 70(1):201-207

6. Cohen PL, et al: Delayed apoptotic cell clearance and lupus-like autoimmunity in mice lacking the c-mer membrane tyrosine kinase. $J$ Exp Med 2002, 196(1):135-140.

7. Navratil JS, Ahearn JM: Apoptosis, clearance mechanisms, and the development of systemic lupus erythematosus. Curr Rheumatol Rep 2001, 3(3):191-198.

8. Huggins ML, et al: Antibodies from systemic lupus erythematosus (SLE) sera define differential release of autoantigens from cell lines undergoing apoptosis. Clin Exp Immunol 1999, 118(2):322-328.

9. Robak E, et al: Peripheral blood lymphocyte apoptosis and circulating dendritic cells in patients with systemic lupus erythematosus: correlation with immunological status and disease-related symptoms. Clin Rheumatol 2006, 25(2):225-233

10. Xie $M$, et al: [Preliminary study on apoptosis and expression of apoptosis-related genes in peripheral blood lymphocytes of patients with systemic lupus erythematosus]. Hua Xi Yi Ke Da Xue Xue Bao 1999, 30(2):192-195.

11. Bombardier C, et al: Derivation of the SLEDAl. A disease activity index for lupus patients. The Committee on Prognosis Studies in SLE. Arthritis Rheum 1992, 35(6):630-640.

12. Fox Rl, et al: Sjogren's syndrome. Proposed criteria for classification. Arthritis Rheum 1986, 29(5):577-585

13. Wallace DJ, et al: A phase II, randomized, double-blind, placebo-controlled dose-ranging study of belimumab in patients with active systemic lupus erythematosus. Arthritis Rheum 2009, 61(9):1168-1178.

14. Koester SK, et al: Monitoring early cellular responses in apoptosis is aided by the mitochondrial membrane protein-specific monoclonal antibody APO2.7. Cytometry 1997, 29(4):306-312.

15. Carthy $\mathrm{CM}$, et al: Early release of mitochondrial cytochrome $\mathrm{c}$ and expression of mitochondrial epitope 7A6 with a porphyrin-derived photosensitizer: $\mathrm{BCl}-2$ and $\mathrm{BCl}-\mathrm{xL}$ overexpression do not prevent early mitochondrial events but still depress caspase activity. Lab Invest 1999, 79(8):953-965

16. Soruri A, et al: Mycobacterial antigens induce apoptosis in human purified protein derivative-specific alphabeta T lymphocytes in a concentration-dependent manner. Immunology 2002, 105(2):222-230.

17. Belot $A$, et al: Protein kinase $C$ delta deficiency causes mendelian systemic lupus erythematosus with B-cell defective apoptosis and hyperproliferation. Arthritis Rheum 2013, 65:2161-2171.
18. Dhir $\mathrm{V}$, et al: Increased T-lymphocyte apoptosis in lupus correlates with disease activity and may be responsible for reduced T-cell frequency: a cross-sectional and longitudinal study. Lupus 2009, 18(9):785-791.

19. Caricchio R, Cohen PL: Spontaneous and induced apoptosis in systemic lupus erythematosus: multiple assays fail to reveal consistent abnormalities. Cell Immunol 1999, 198(1):54-60.

20. Liu C, et al: Effects of sex hormones on apoptosis in peripheral blood mononuclear cells from patients with systemic lupus erythematosus. Chin Med J (Engl) 2001, 114(3):291-293.

21. Bengtsson AA, et al: Induction of apoptosis in monocytes and lymphocytes by serum from patients with systemic lupus erythematosus - an additional mechanism to increased autoantigen load? Clin Exp Immunol 2004, 135(3):535-543.

22. Armstrong DJ, et al: Distinctive effects of G-CSF, GM-CSF and TNFalpha on neutrophil apoptosis in systemic lupus erythematosus. Clin Exp Rheumatol 2005, 23(2):152-158.

23. Chen W, Lin J: Lymphopenia relating to T-lymphocyte apoptosis in systemic lupus erythematosus. Clin Rheumatol 2011, 30(11):1515-1516.

24. Munoz LE, et al: Apoptosis in the pathogenesis of systemic lupus erythematosus. Lupus 2008, 17(5):371-375

25. Manson JJ, Isenberg DA: The origin and pathogenic consequences of anti-dsDNA antibodies in systemic lupus erythematosus. Expert Rev Clin Immunol 2006, 2(3):377-385.

26. Dillon CF, Jones JV, Reichlin M: Antibody to Ro in a population of patients with systemic lupus erythematosus: distribution, clinical and serological associations. J Rheumatol 1983, 10(3):380-386.

27. Villarreal GM, et al: Prevalence of 13 autoantibodies and of the $16 / 6$ and related pathogenic idiotypes in $\mathbf{4 6 5}$ patients with systemic lupus erythematosus and their relationship with disease activity. Lupus 1997, 6(5):425-435.

28. Carthy $\mathrm{CM}$, et al: $\mathrm{BCl}-2$ and $\mathrm{BCl}-\mathrm{xL}$ overexpression inhibits cytochrome $\mathrm{C}$ release, activation of multiple caspases, and virus release following coxsackievirus B3 infection. Virology 2003, 313(1):147-157.

29. Pan Y, et al: [Effects of glucocorticoids on the expression of GTIR and apoptosis of the CD4(+)CD25(+)CD127(dim/-) T cells in patients with systemic lupus erythematosus]. Beijing Da Xue Xue Bao 2012, 44(2):215-220.

30. Bohm I: Apoptosis: the link between autoantibodies and leuko-/lymphocytopenia in patients with lupus erythematosus. Scand J Rheumatol 2004, 33(6):409-416.

31. Linker-Israeli M, Quismorio FP Jr, Horwitz DA: CD8+ lymphocytes from patients with systemic lupus erythematosus sustain, rather than suppress, spontaneous polyclonal lgG production and synergize with CD4+ cells to support autoantibody synthesis. Arthritis Rheum 1990, 33(8):1216-1225.

32. Midgley A, et al: The role of neutrophil apoptosis in juvenile-onset systemic lupus erythematosus. Arthritis Rheum 2009, 60(8):2390-2401.

33. Habib HM, et al: Enhanced propensity of T lymphocytes in patients with systemic lupus erythematosus to apoptosis in the presence of tumour necrosis factor alpha. Scand J Rheumatol 2009, 38(2):112-120.

34. Balanescu $E$, et al: Immunohistochemical aspects of apoptosis in subcutaneous lupus erythematosus. Rom J Intern Med 2010, 48(3):261-265.

35. Makino $\mathrm{H}$, et al: Glomerular cell apoptosis in human lupus nephritis. Virchows Arch 2003, 443(1):67-77.

doi:10.1186/1479-5876-11-261

Cite this article as: Su et al:: The association among leukocyte apoptosis, autoantibodies and disease severity in systemic lupus erythematosus. Journal of Translational Medicine 2013 11:261 